\title{
Deep Learning Algorithms Correctly Classify Brassica rapa Varieties Using Digital Images
}

\begin{abstract}
Minah Jung ${ }^{1,2+}$, Jong Seob Song ${ }^{2 \dagger}$, Seongmin Hong ${ }^{3,4}$, SunWoo Kim ${ }^{5}$, Sangjin Go ${ }^{3,6}$, Yong Pyo Lim ${ }^{4}$, Juhan Park ${ }^{2}$, Sung Goo Park ${ }^{1,7}$ and Yong-Min Kim ${ }^{3 *}$

${ }^{1}$ Department of Functional Genomics, KRIBB School of Biological Science, Korea University of Science and Technology, Daejeon, South Korea, ${ }^{2}$ Euclidsoft Co., Ltd, Daejeon, South Korea, ${ }^{3}$ Genome Editing Research Center, Korea Research Institute of Bioscience and Biotechnology, Daejeon, South Korea, ${ }^{4}$ Molecular Genetics and Genomics Laboratory, Department of Horticulture, College of Agriculture and Life Science, Chungnam National University, Daejeon, South Korea, ${ }^{5}$ Department of Bio-Al Convergence, Chungnam National University, Daejeon, South Korea, ${ }^{6}$ Department of Horticulture, Gyeongsang National University, Jinju, South Korea, ${ }^{7}$ Disease Target Structure Research Center, Korea Research Institute of Bioscience and Biotechnology, Daejeon, South Korea
\end{abstract}

Efficient and accurate methods of analysis are needed for the huge amount of biological data that have accumulated in various research fields, including genomics, phenomics, and genetics. Artificial intelligence (Al)-based analysis is one promising method to manipulate biological data. To this end, various algorithms have been developed and applied in fields such as disease diagnosis, species classification, and object prediction. In the field of phenomics, classification of accessions and variants is important for basic science and industrial applications. To construct Al-based classification models, three types of phenotypic image data were generated from 156 Brassica rapa core collections, and classification analyses were carried out using four different convolutional neural network architectures. The results of lateral view data showed higher accuracy compared with top view data. Furthermore, the relatively low accuracy of ResNet50 architecture suggested that definition and estimation of similarity index of phenotypic data were required before the selection of deep learning architectures.

Keywords: artificial intelligence, deep learning, classification model, phenotypic analysis, Brassica rapa (Brassicaceae)

\section{INTRODUCTION}

One of the major features of modern science is convergent analyses using heterogeneous technologies from multiple and independent fields to analyze huge amounts of data. To manipulate these data, artificial intelligence (AI) technology has come into the spotlight. Deep learning is a type of AI that uses computer algorithms based on artificial neural networks (ANNs), which mimic the principles and structure of human neural networks to emulate human cognitive processes (Chauhan et al., 2018). In an ANN, artificial neurons (nodes) combine synapses to form a network and strengthen synapses through learning, thus acquiring problem-solving capabilities. An ANN consists of three major components: an input layer that receives data, an output layer that presents the results of analysis, and hidden layers that exist between the input and output layers. To construct an analytic model that uses deep learning, the numbers of nodes and hidden layers must be specified. 
Research has shown that the outcome of machine learning can be improved by increasing the number of hidden layers in the model. A machine learning method with two or more hidden layers is referred to as a deep neural network (DNN).

An ANN consisting only of a single fully connected layer, called a "fully connected neural network," is usually used for image analysis with one-dimensional input data, which requires the dimensionality of the data to be reduced from three to one. This results in a lack of information for the AI neural network to use in extracting and learning features, resulting in limited accuracy. To overcome the limitations of the fully connected neural network, a different model called the convolutional neural network $(\mathrm{CNN})$ was developed for the analysis of image or video data. A CNN consists of two layers: a convolutional layer and a pooling layer (Lecun et al., 1998). The convolutional layer is a prerequisite that reflects the activation function after applying a filter to the input data. The pooling layer is used to reduce the size of the activation map or to highlight specific data. Then, features of images were extracted through sequential analyses of two layers, namely, convolution and pooling layers. The fully connected neural network, the $\mathrm{CNN}$, maintains the dimensions of the image data in each layer. For image analyses using deep learning, new CNN architectures are developed every year and presented in the ImageNet Large Scale Visual Recognition Competition. Thus, year after year, errors are reduced and accuracy is increased by changing the layer composition, depth, and calculation methods used in CNNs. In previous studies, several applications of CNN architectures showed outstanding results in the competition in the past decade (Dhaka et al., 2021) such as AlexNet (Krizhevsky et al., 2012), VGG19 (Simonyan and Zisserman, 2014), Inception v3 (Szegedy et al., 2016), Inception v4 (Szegedy et al., 2017), GoogLeNet (Szegedy et al., 2015), and ResNet50 (He et al., 2016), DenseNet121 (Huang et al., 2017), and SqueezeNet (Iandola et al., 2016).

As AI research becomes more popular, applications of AI have rapidly expanded to various research fields. In biology, AI-based analysis is used for detection, classification, and recognition with genomic and phenotypic data from humans, animals, and plants. In human research, AI-based approaches are used to classify pathogens into genetic subgroups (Prajapati et al., 2017; Sardogan et al., 2018), distinguish patient groups with different risk factors, and detect objects in images that can be used for diagnosis (Ubbens et al., 2018; Jiang et al., 2020). Animal data are also used to classify or diagnose diseases (Banzato et al., 2018a,b; Choi et al., 2018; Kim et al., 2019) and to study animal cognition (Hao et al., 2019; Yudin et al., 2019; Mohammed and Hussain, 2021). In plants, AI-based image analyses can be used to recognize specific tissues (i.e., flowers and fruits), detect diseases (Wozniak and Połap, 2018; Maeda-Gutierrez et al., 2020), and classify species, cultivars, and lineages (Lee et al., 2015; Grinblat et al., 2016; Hedjazi et al., 2017).

Plant classification plays important roles in the preservation of biodiversity, maintenance of economically important crops for food security, and discovery of new therapeutic substances, such as Tamiflu ${ }^{\circledR}$ from star anise (Illicium verum) and Artemisinin from sweet wormwood (Artemisia annua) (Ingram and Porter, 2015). The classification of plant accessions or species was traditionally carried out by grow-out tests based on phenotypes or morphologies. In recent decades, an explosion in nextgeneration sequencing capabilities has led to the widespread use of genetic information to classify plants. AI-based technologies now have the potential to revolutionize basic plant science, as well as breeding programs, by allowing rapid, noninvasive identification of plant varieties on the basis of digital images that can be easily acquired in high volume and at low cost.

We used four different CNN architectures to construct deep learning models to classify accessions from the Brassica rapa core collection on the basis of digital images. Each accession belongs to one of four groups in the core collection: Chinese accessions, early introduced accessions, Korean breeding accessions, and non-pekinensis accessions. The task of the deep learning models was to assign individual plants to the correct group using data from a single image. Four image datasets of 156 different accessions were generated. The first three datasets consisted of images taken from above the plants (top view), whereas the fourth dataset consisted of images taken from the side of the plants (lateral view). Each dataset was divided into a training set and a test set, and classification models were constructed using the AlexNet, VGG19, GoogLeNet, and ResNet50 architectures with over 50 iterations with randomly chosen data from the training sets. The results showed that the accuracy was generally higher for the lateral view images than for the top view images. Comparisons among the four architectures revealed that the GoogLeNet and VGG19 architectures had the highest accuracy with the top view images and the lateral view images, respectively, whereas the ResNet50 architecture had the lowest accuracy regardless of the dataset used.

\section{METHODS}

\section{Plant Materials and Generation of Plant Images}

We used 156 lines of the B. rapa core collection to produce three datasets for the development of classification models based on the morphology of Chinese cabbage (Pang et al., 2015). The individual lines in the core collection are classified as Chinese accessions, early introduced accessions, Korean breeding accessions, or non-pekinensis accessions depending on their geographic origin (Figure 1). The Chinese accessions include species native to China. The early introduced accessions are a group of lines that were imported to Korea in the early 1900s. The Korean breeding accessions are lines that are currently used by breeding companies in Korea. The non-pekinensis accessions comprise various subspecies of $B$. rapa including oil seed types, bok choy, turnip, and others. All accessions were cultivated in trial fields at the Chungnam National University from 2018 to 2021 to generate the top view and lateral view images.

Images of individual plants were generated with a digital single-lens reflex camera (Nikon D5300, 18-55 mm VR II). To create lateral view images of heading traits, plants were grown for 10 weeks in trial fields with 10 replicates per accession in 2018. Ten individuals per accession were then harvested, and a representative individual was selected to generate lateral view 


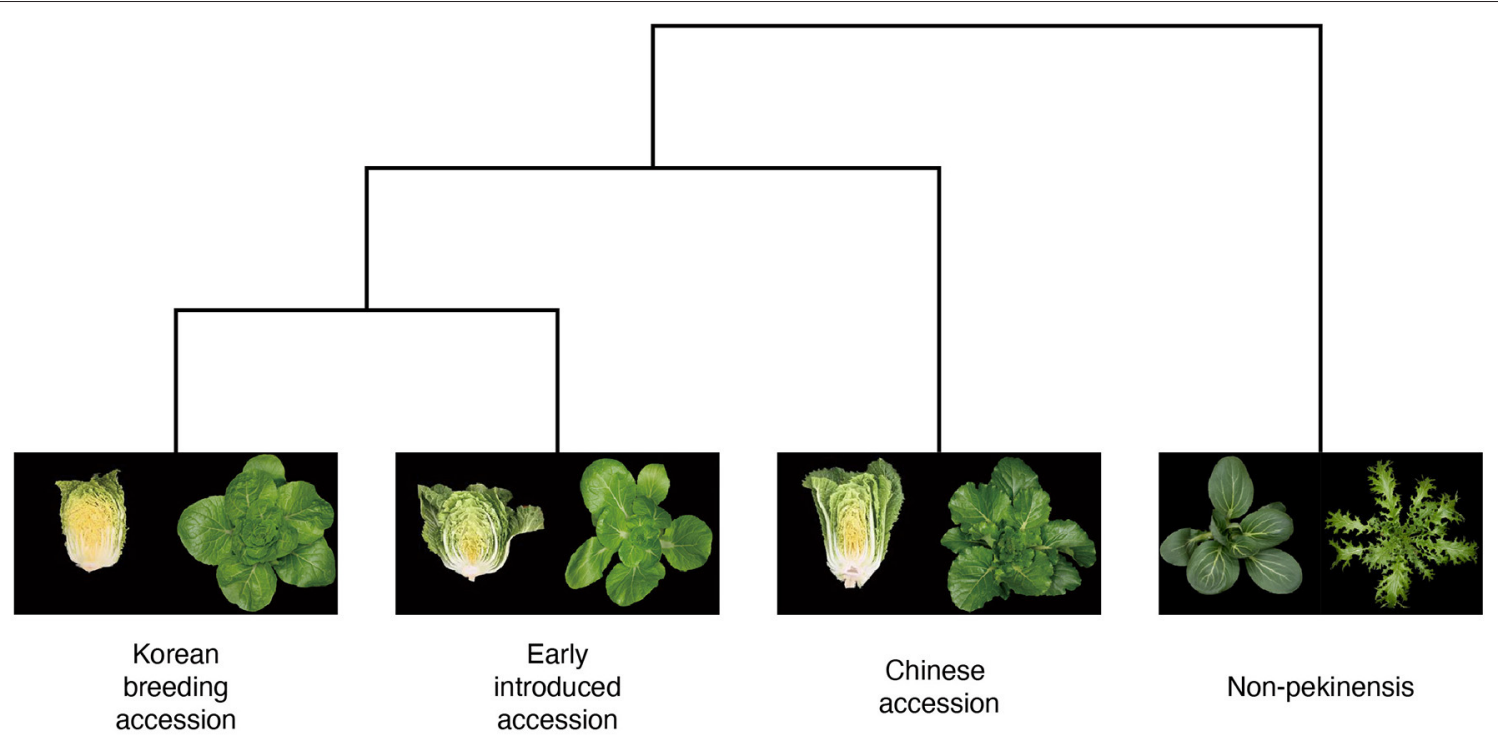

FIGURE 1 | Phylogenetic tree of Brassica rapa with phenotypic images. The phylogenetic tree shows breeding history of four different groups (Korean breeding accession, early introduced accession, Chinese accession, and non-pekinensis) from Chinese cabbage in the east Asia (Ramchiary et al., 2011). Image data were generated from top (four groups) and lateral views (three groups) of plants from the B. rapa core collections. The trait of forming leafy head is the main factor to distinguish subspecies pekinensis.

images. The images were photographed from the cross-sectional side of Chinese cabbages forming leafy heads. To generate top view images, we considered environmental conditions for core collection. The core collection contained various growing conditions such as spring-, summer-, autumn-, and winter(southern part of Korea) harvest phenotypes. Thus, we grew the core collection in two conditions: (1) from autumn in 2020 (top views 1 and 2) and (2) from spring to summer in 2021 (top view 3). In total, five plants per accession of core collection were grown in the green house to maintain the same growth condition. Images were generated at the end of the 1st (2021), 7th (2020), and 9th (2020) week after planting using a customized photograph booth to provide the same light condition by blocking external light. Obtained phenotype data from the 7th and 9th week were grouped as top views 1 and 2 , respectively. Then, phenotype data from the 1 st week were grouped as top view 3. Among the 156 accessions, a total of three accessions, one Chinese and two non-pekinensis, could not be germinated and were excluded. In addition, three accessions showed an early flowering phenotype and were also excluded. In total, 2,138 images of 150 accessions were used for analysis (Table 1).

\section{Preparation of Datasets for the Image Classification Models}

Before analysis, the background of the subject in each image was erased for effective identification. Next, the images were resized to 224 pixels, which is a size commonly used in CNN analysis (Zeiler and Fergus, 2014). Then, to obtain more images for the training model, data augmentation was performed using rotation by $90^{\circ}, 180^{\circ}$, and $270^{\circ}$ (Shorten and Khoshgoftaar, 2019)
(Supplementary Figure 1). In the case of the lateral view dataset, we had more rotation augmentation with $+/$ - 10 degrees because of the relatively small sample size. Each dataset was divided into a training set and a test set with a sample size ratio of 8-2 between the two sets (Step 1 in Figure 2). The initial training set was further divided into a final training set and a validation set, again with a sample size ratio of $8-2$ between the two sets (Step 2 in Figure 2). The final training set was used to construct the models, and the validation set was used to advance the model through hyperparameter tuning.

\section{Application of the Four Pretrained CNN Models}

We used four pretrained CNN models, namely, AlexNet, VGG19, GoogLeNet, and ResNet50, to classify each accession on the basis of phenotypic image data (Figure 3). Each architecture has characteristics that distinguish it from the others. AlexNet is composed of five convolutional layers and three fully connected layers and uses a "dropout" function to avoid overfitting by switching off certain neurons. It performs parallel computation with two graphics processing units (GPUs). VGG19 consists of 19 layers and uses a large $3 \times 3$ kernel size filter, which can increase the depth of the network. GoogLeNet comprises 22 layers and has an "inception module" consisting of $1 \times 1,3 \times 3$, and $5 \times 5$ convolutions, which enables dimensionality reduction. ResNet50 creates an identity block with a shortcut connection that skips one or more layers on the basic multilayer structure. The analysis performances of CNN architectures, such as GoogLeNet and VGG19 with deep layers, were better than those with fewer layers like AlexNet (Table 2). However, constructing too many layers is inefficient because it takes much time and effort to calculate. 
TABLE 1 | Datasets used for analysis.

\begin{tabular}{|c|c|c|c|c|}
\hline & \multicolumn{4}{|c|}{ Accessions (accessions/images) } \\
\hline & Chinese & Korean breeding & Early introduced & Non-pekinensis \\
\hline Top view 1 & $45 / 220$ & $54 / 266$ & $33 / 164$ & $18 / 86$ \\
\hline Top view 2 & $35 / 171$ & $53 / 250$ & $30 / 144$ & $4 / 16$ \\
\hline Top view 3 & $47 / 235$ & $53 / 265$ & $30 / 150$ & $13 / 65$ \\
\hline Lateral view & $25 / 51$ & $25 / 37$ & $9 / 18$ & $-/-$ \\
\hline Total & $47 / 677$ & $54 / 818$ & $33 / 476$ & $18 / 167$ \\
\hline
\end{tabular}

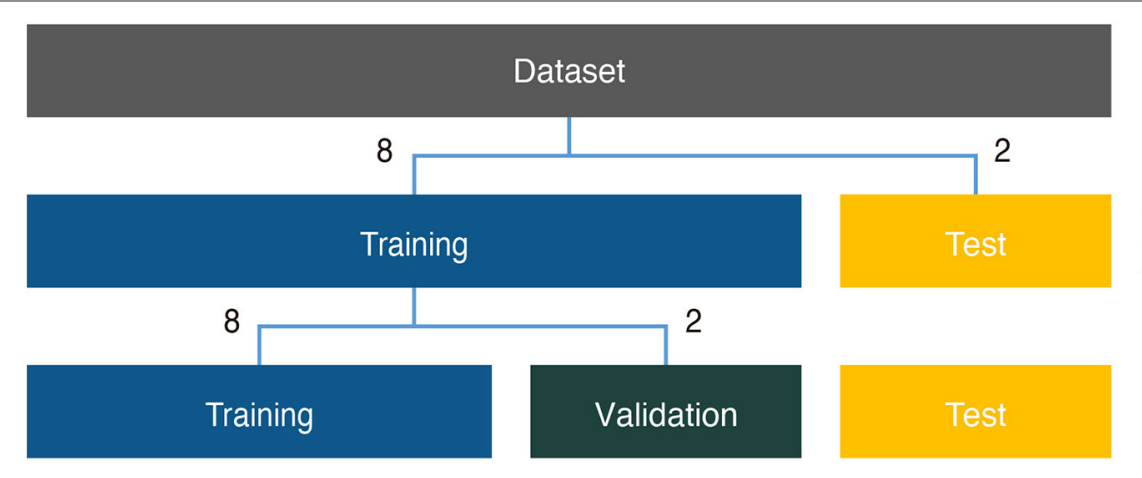

Step 1.

Split out Test Set

for Model Evaluation

Step 2.

Division of Training Set for Model

Construction and Validation

FIGURE 2 | Analysis scheme for phenotypic classification. Each dataset was divided into training, validation, and test sets for construction of classification models. The size ratio of the training and test sets was 8:2 for the model evaluation (Step 1). The training set was subdivided at an 8:2 size ratio for the model construction and validation (Step 2).

Among pretrained models with more than a certain number of layers, a model with a large number of hyperparameters such as ResNet50 has good analysis performance. If precise tuning of hyperparameters was involved, the accuracy was relatively higher than that of the model with deeper layers (Dhaka et al., 2021).

\section{Improvement of the Models Through Hyperparameter Tuning}

To optimize each pretrained model for each dataset (top view or lateral view), we tuned the hyperparameters by changing the learning rate and optimizer. The batch size was determined based on the sample size of each dataset. "Softmax" was used as an activation function, which is a common practice in $\mathrm{CNN}$ analysis. Two optimizers were considered, "A Method for Stochastic Optimization" (ADAM) (Kingma and Adam, 2018) and "Stochastic Gradient Descent" (SGD) (Bottou, 2010). "Categorical Crossentropy," which is known to be suitable for classification, was used as a loss function to reduce loss, or the difference between actual and predicted values. To prevent overfitting, an "earlystopping" function that stops the training of the model under certain conditions was applied using the loss of the validation set.

Each model was tested with 50 iterations using the training set with random sampling and replacement. For each architecture, the model showing the highest accuracy after validation was selected as the final classification model. Then, the final classification models were tested using the test datasets, which were not used at all in the construction of the classification model. Four pretrained models were created with python (interface) and keras (framework). In addition, all analyses were executed using a Tesla V100 GPU with 32 GB video random access memory (VRAM) and a 112 core process central processing unit (CPU).

\section{RESULTS}

\section{Construction of the Deep Learning Classification Models}

We carried out $\mathrm{CNN}$ analysis using the four pretrained models and four different image datasets (three top view and one lateral view). To implement a model suitable for each dataset, we tuned hyperparameters such as batch size, optimizer, epoch, and learning rate by iterative validation. The batch size was tuned according to the sample size of the dataset, resulting in a batch size of 2 for the top view datasets and 17 for the lateral view datasets. SGD was chosen as the optimizer for all models and datasets except for the ResNet50 architecture with the top view 1 dataset, for which ADAM was selected. The learning rate was adjusted according to overshooting or learning time, and an appropriate rate from 0.0001 to 0.001 was set for each dataset and architecture. The epoch was set to 500 for all datasets (Table 3). 


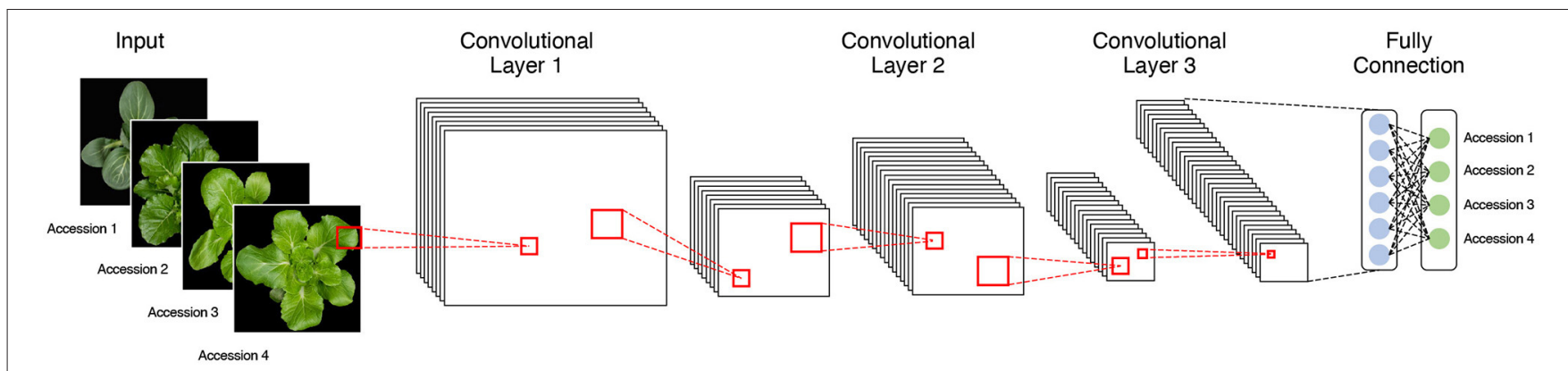

FIGURE 3 | The basic structure of a convolutional neural network (CNN) analysis with image data as input. The classification model is composed of multiple convolution and pooling layers.

TABLE 2 | Features of the four pretrained models.

\begin{tabular}{|c|c|c|c|}
\hline Architecture & Year & \# Layers (Convolutional + fully connected) & Model description \\
\hline LeNet-5 & 1998 & $7(5+2)$ & Introduction of CNN Concepts \\
\hline AlexNet & 2012 & $8(5+3)$ & Parallel computing with 2 GPUs \\
\hline VGG19 & 2014 & $19(16+3)$ & Multiple layers \\
\hline GoogLeNet & 2014 & $22(21+1)$ & Inception module \\
\hline ResNet50 & 2015 & $50(49+1)$ & Skip connection \\
\hline SqueezeNet & 2016 & Squeeze + Expand layers & Lightweight model with small size \\
\hline Inception v3 & 2016 & $52(42+10)$ & Improvement from GoogLeNet \\
\hline Inception v4 & 2017 & 75 convolution layers & More layers with better performance rather than inception v3 \\
\hline DenseNet-121 & 2017 & 117 convolution +3 transition +1 classification layers & Connected with all layers \\
\hline
\end{tabular}

TABLE 3 | Optimized hyperparameter for model advancement.

\begin{tabular}{|c|c|c|c|c|}
\hline \multirow[t]{2}{*}{ Hyperparameter } & \multicolumn{4}{|c|}{ Value } \\
\hline & ResNet50 & AlexNet & GoogLeNet & VGG19 \\
\hline \multirow[t]{2}{*}{ Batch size } & \multicolumn{4}{|c|}{ Top: 2} \\
\hline & \multicolumn{4}{|c|}{ Lateral: 17} \\
\hline Activation & \multicolumn{4}{|c|}{ Softmax } \\
\hline Optimizer & ADAM & SGD & SGD & SGD \\
\hline \multirow[t]{4}{*}{ Learning rate } & Top 1: 1e-4 & Top 1: 1e-5 & Top 1: 1e-4 & Top 1: 1e-3 \\
\hline & Top 2: 1e-5 & Top 2: $5 \times 1 e-4$ & Top 2: 1e-4 & Top 2: $5 \times 1 e-4$ \\
\hline & Top 3: 1e-4 & Top 3: 1e-4 & Top 3: 1e-4 & Top 3: $5 \times 1 e-3$ \\
\hline & Lateral:1e-4 & Lateral:1e-4 & Lateral: $5 \times 1 \mathrm{e}-4$ & Lateral:1e-4 \\
\hline Epoch & \multicolumn{4}{|c|}{500} \\
\hline Early stopping & \multicolumn{4}{|c|}{5} \\
\hline Loss function & \multicolumn{4}{|c|}{ Categorical cross-entropy } \\
\hline
\end{tabular}

\section{Classification of Individual Top View Images Using the CNN Models}

A classification model was designed for each of three independent top view datasets (top view 1, top view 2, and top view $3)$. For the top view 1 dataset, the average classification accuracy based on 50 iterative validations was $43.44 \%$. The minimum accuracy was $26.77 \%$ with ResNet50, and the maximum accuracy was $64.06 \%$ with GoogLeNet (Figure 4 and Supplementary Table 1). A model showing high accuracy for each architecture was selected as the final classification model (Supplementary Figure 2). Evaluation of the final classification model using 154 images in the test set showed accuracies of 45.64\% (AlexNet), 46.98\% (VGG19), 49.66\% (GoogLeNet), and $37.58 \%$ (ResNet50) (Figure 4). Whereas the classification for non-pekinensis accessions and Korean breeding accessions was accurate regardless of the architecture, Chinese accessions often varied depending on the architecture, and most cases of misclassification were classified as Korean breeding accession. In 


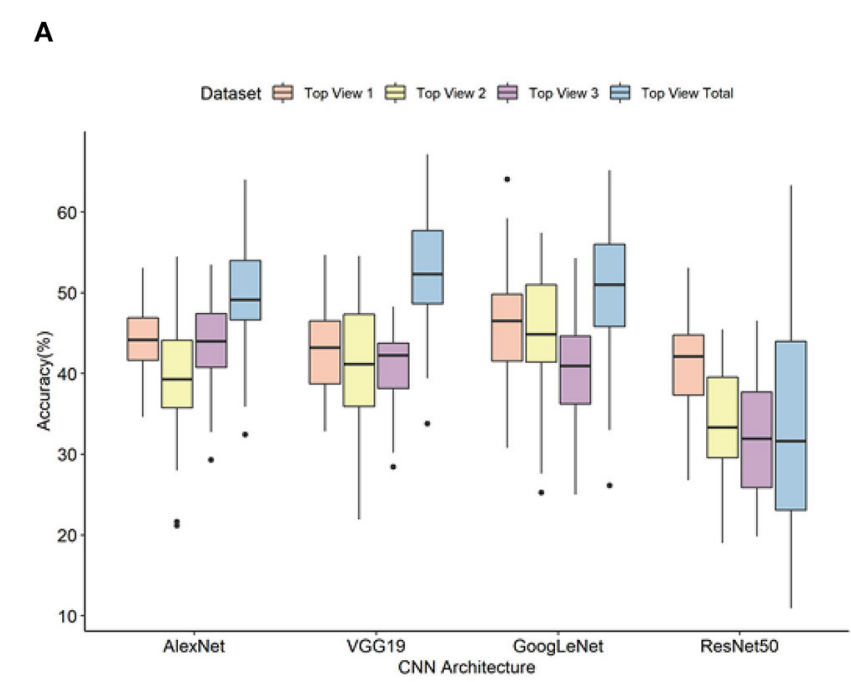

\section{B}

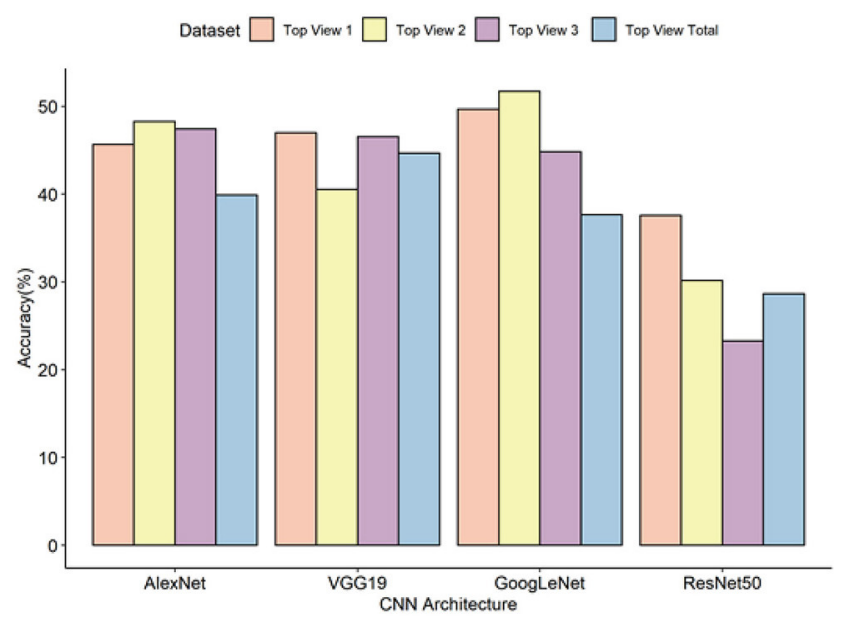

C

Dataset 追 Lateral View 追 Top View Total

D
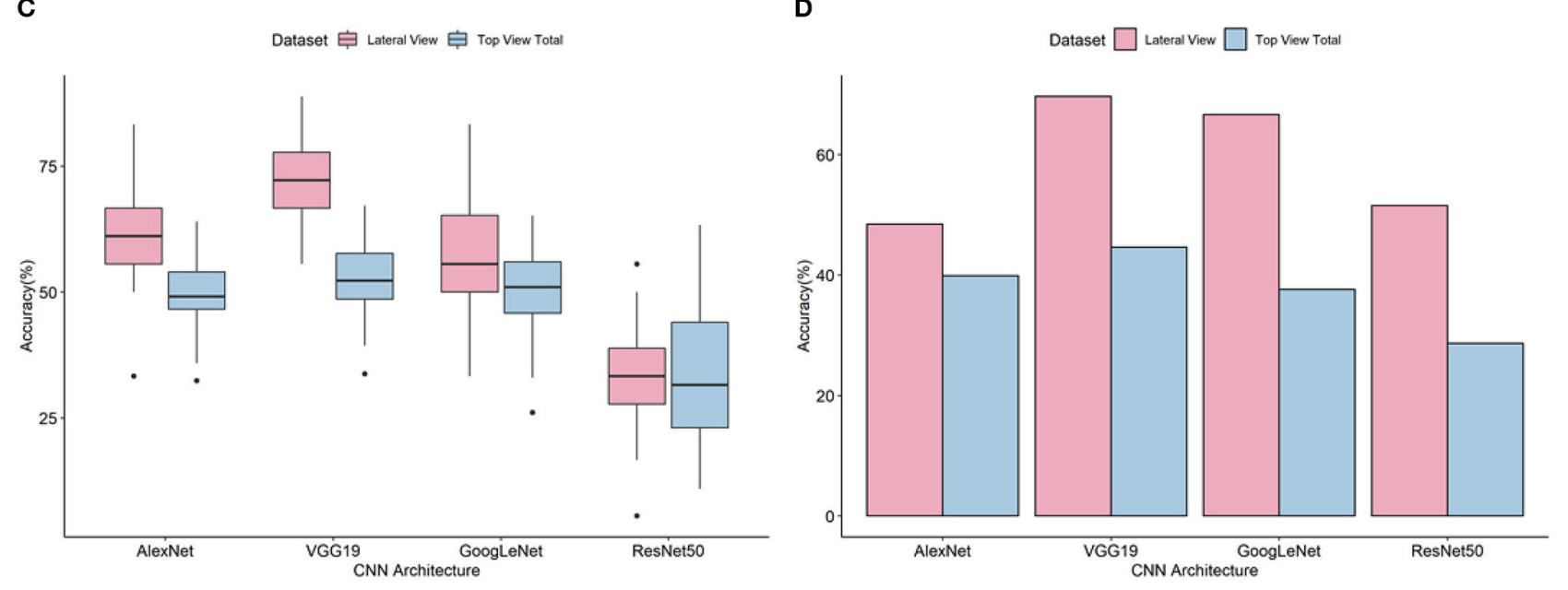

FIGURE 4 | Validation and evaluation of the classification models.(A) Prediction accuracy of top view datasets based on 50 iterative validations. (B) Prediction accuracy using the test set of top view datasets. (C) Prediction accuracy of top and lateral view datasets based on 50 iterative validations. (D) Prediction accuracy using the test set of top and lateral view datasets. The horizontal axis indicates the analytical architectures, and the vertical axis indicates the prediction accuracy (\%). Orange, yellow, purple, light blue, and pink colors stand for types of image dataset.

case of early introduced accession, the prediction performance was the lowest, and it was misclassified into Korean breeding accession or Chinese accession (Supplementary Table 2).

For the top view 2 dataset, the average prediction accuracy based on 50 iterative validations was $39.78 \%$. The minimum accuracy was $19.00 \%$ with ResNet50, and the maximum was 57.43\% with GoogLeNet (Figure 4 and Supplementary Table 3). Evaluation of the final classification model using 116 images in the test set showed accuracies of $48.28 \%$ (AlexNet), 40.52\% (VGG19), 51.72\% (GoogLeNet), and 30.17\% (ResNet50) (Figure 4 and Supplementary Figure 3). The classifications for Chinese accessions and early introduced accessions varied among the different architectures. For the Chinese accessions, accuracy differences of more than two times were shown in accordance with architecture. According to the CNN architecture, the classification test accuracy of early introduced accession of the model was large difference until 31.03\%, and most misclassified cases were classified as Korean breeding accession or Chinese accession (Supplementary Table 4).

For the top view 3 dataset, the average prediction accuracy based on 50 iterative validations was $41.06 \%$. The minimum accuracy was $13.13 \%$ with ResNet50, and the maximum was 60.19\% with GoogLeNet (Figure 4 and Supplementary Table 5). Evaluation of the final classification model using 116 images in the test set showed accuracies of $47.41 \%$ (AlexNet), 46.55\% (VGG19), 44.83\% (GoogLeNet), and 23.28\% (ResNet50) (Figure 4 and Supplementary Figure 4). Among them, test sets from the group of Chinese accession were misclassified as the group of Korean breeding accession or group of early introduced accession. In addition, classification errors classified as the group of Chinese accession or Korean breeding were also occurred in the group of early introduced accession. Like top views 1 
and 2, top view 3 dataset also showed high differences among architectures shown as top views 1 and 2 in the group of Chinese and early introduced accession while Korean breeding accessions were correctly classified regardless of architectures (Supplementary Table 6).

\section{Classification of Whole Top View Images Using the CNN Models}

To confirm performance of classification for the top view phenotypic images regardless of developmental stages, classification analysis of the top view dataset was performed using all of the top view data including top view 1, 2, and 3 . For all of the top view dataset, the average prediction accuracy based on 50 iterative validations was $37.46 \%$. The minimum accuracy was $18.26 \%$ with ResNet50, and the maximum was 51.69\% with VGG19 (Figure 4 and Supplementary Table 7). Model with highest validation accuracy, 64.00, 67.16, 65.17 and $63.29 \%$ was selected as the final classification model, each architecture respectively.. Evaluation of the final classification model using 116 images in the test set showed accuracies of 39.89\% (AlexNet), 44.66\% (VGG19), 37.64\% (GoogLeNet), and $31.74 \%$ (ResNet50) (Figure 4 and Supplementary Figure 5). As more data were acquired, it is encouraging that nonpekinensis classification accuracy increases. In individual datasets, it was difficult to classify non-pekinensis due to the small number of data available for model construction and testing, but more numbers of data gathered together and the prediction increased to non-pekinensis. Similar to individual top view datasets, the Korean breeding accession was best predicted with high accuracy, followed by Chinese accession. Early introduced accession was incorrectly classified as Korean breeding access or Chinese accession in all models except ResNet50 (Supplementary Table 8).

Furthermore, a combination of training set and test set was designed to investigate classification accuracy by developmental stages (Supplementary Table 9). For example, similar developmental stages, top views 1 and 2, were used as training set and early developmental stage, top view 3, was used as test set. Accuracy of test set in top view 1 was predicted as $44.12 \%$ (Supplementary Table 9), and top view 2 was predicted as the accuracy of $46.00 \%$ (Supplementary Table 10). However, top view 3 was predicted as the accuracy of 36.82\% (Supplementary Table 11). These results suggested that phenotypes of core collection in early developmental stages were different from those of mature stages and that similar developmental stages were required to construct a classification model in early developmental stages.

\section{Classification of Lateral View Images Using the CNN Models}

For the lateral view dataset, the deep learning analysis was carried out with $\pm 10^{\circ}$ rotation augmentation. In addition, no lateral view images of the non-pekinensis accessions were generated. Therefore, the deep learning analysis was carried out using images of only three groups. The classification accuracy based on the validation results ranged from 13.04 to $88.89 \%$, with an average of $58.34 \%$ (Figure 4 and Supplementary Table 12).
Evaluation of the final classification model with the test images showed classification accuracies of $48.48 \%$ (AlexNet), 69.70\% (VGG19), 66.67\% (GoogLeNet), and 51.52\% (ResNet50) (Figure 4 and Supplementary Figure 6). Both the Korean breeding accessions and the Chinese accessions were well classified. For the Chinese accessions, 9 of the 16 test images were correctly classified with all the architectures. In the case of the early introduced accessions, three test images were classified incorrectly by all of the architectures (Supplementary Table 13).

To compare the classification accuracy of lateral and top view datasets, classification of top view 2 dataset except non-pekinensis was carried out using four architectures. Top view 2 and lateral view datasets were generated at a similar developmental stage. The average prediction accuracies of the lateral view dataset based on 50 iterative validations were 52.00, 63.27, 65.22, and 59.57\% from AlexNet, VGG19, GoogLeNet, and ResNet50, respectively. The evaluation of the top view 2 (three groups) classification model showed accuracies of 46.43\% (AlexNet), 55.36\% (VGG19), 64.29\% (GoogLeNet), and $37.50 \%$ (ResNet50). For all architectures, the accuracy of lateral view was higher than that of top view classification results (Supplementary Figure 7).

\section{Comparison of CNN Architecture Performances}

Final classification models were constructed using the four different pretrained $\mathrm{CNN}$ architectures. The accuracies of classification for the test set of the top view 1 dataset were $45.64 \%$ (AlexNet), 46.98\% (VGG19), 49.66\% (GoogLeNet), and 51.52\% (ResNet50). For the top view 2 dataset, the accuracies for the validation set were 48.28\% (AlexNet), 40.52\% (VGG19), 51.72\% (GoogLeNet), and 30.72\% (ResNet50). For the top view 3 dataset, the accuracies for the test set were $47.41 \%$ (AlexNet), $46.55 \%$ (VGG19), 44.83\% (GoogLeNet), and 23.28\% (ResNet50). For the lateral view dataset, the classification accuracies for the test set were 56.94\% (AlexNet), 72.22\% (VGG19), 61.59\% (GoogLeNet), and $41.61 \%$ (ResNet50). The GoogLeNet architecture gave the most accurate classifications for top view 1 and 2 datasets, and lateral view and all top view dataset were classified with high accuracy from the VGG19 model. The highest accuracy for any architecture was $69.70 \%$ for the VGG19 architecture with the lateral view dataset. The highest accuracies for the AlexNet and GoogLeNet architectures with the lateral view dataset were 48.48 and $66.67 \%$, respectively. The ResNet50 architecture showed the lowest accuracies of $37.58,30.17,23.28$, and $28.65 \%$ for the top view 1 , top view 2, top view 3 , and all top view datasets, respectively. These results suggested that ResNet50 was not appropriate for the classification of our B. rapa phenotypes because of its analytical algorithm.

\section{Pairwise Comparisons Between Pairs of Accession Types}

We tested the ability of the final classification model to correctly classify pooled images of pairs of accession types from the top view 1 dataset with each of the four architectures (Figure 5). The non-pekinensis accessions were classified as the most accurate overall in the pairwise tests. The highest accuracy for any pair of 
A

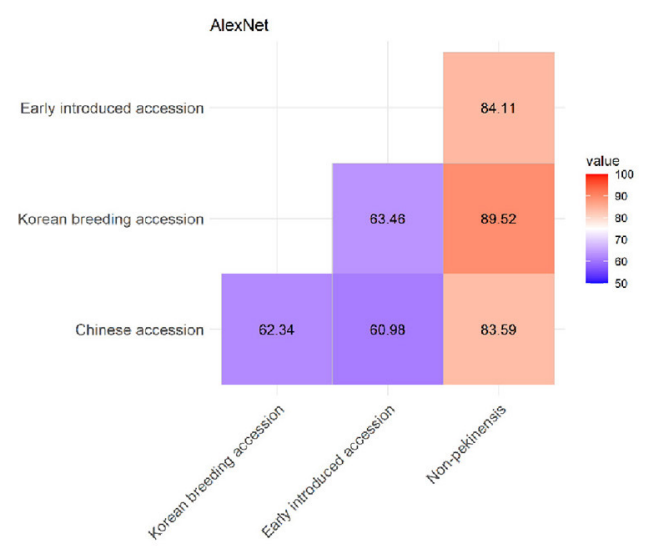

C

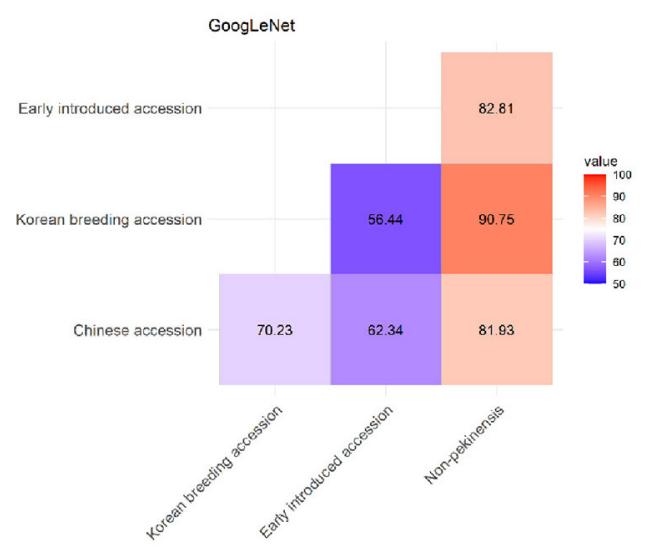

B

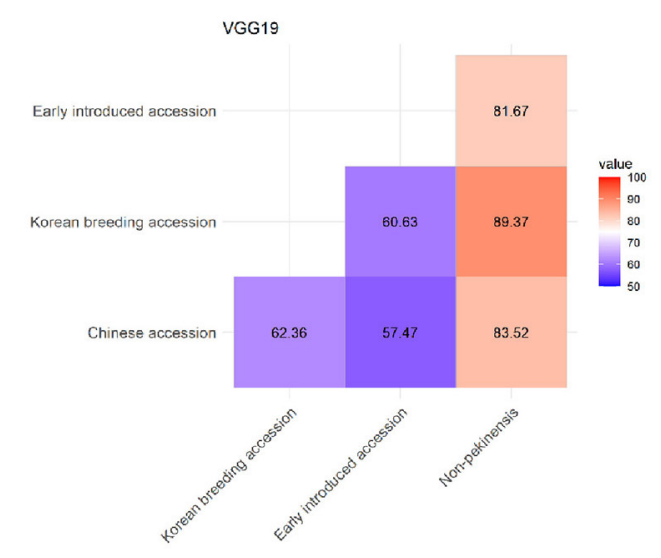

D

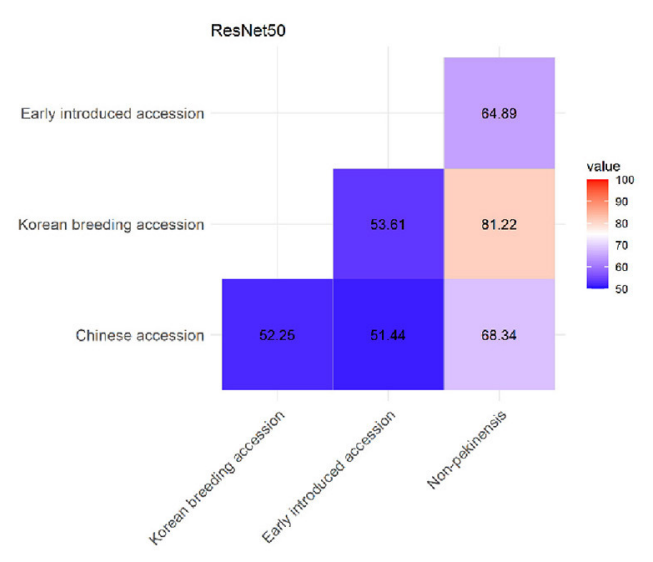

FIGURE 5 | Classification accuracy in pairwise tests using the four types of accessions (Chinese, early introduced, Korean breeding, and non-pekinensis) with four different architectures: (A) AlexNet, (B) VGG19, (C) GoogLeNet, and (D) ResNet50.

accession types was achieved with the non-pekinensis accessions and the Korean breeding accessions, which produced an average accuracy of $87.72 \%$ over 50 iterations and a maximum accuracy of $90.75 \%$ with the GoogLeNet architecture (Figure 5C). The average accuracies achieved with the non-pekinensis accessions and the Chinese and early introduced accessions were 79.34 and $78.37 \%$, respectively. In contrast to the non-pekinensis accessions, the early introduced accessions were relatively poorly classified overall, reaching only $58.54 \%$ accuracy in pairwise tests with the Korean breeding accessions and $61.79 \%$ accuracy in pairwise tests with the Chinese accessions. These results suggest that the early introduced accessions share some phenotypic features with the Korean breeding and Chinese accessions, which led to classification errors.

\section{DISCUSSIONS}

Huge amounts of phenotypic data are accumulating in the biological sciences. As the technology for smart farms advances, the generation and analysis of image-based phenotypic data will play a curial role in agriculture. Hence, the development of classification models using deep learning is important for both basic research and applied science. To this end, we performed deep learning-based classification analyses using images of $B$. rapa. Our results showed that deep learning architectures were able to correctly classify top view images of $B$. rapa plants more accurately at 7 weeks after planting (top view 1 dataset, average accuracy $=43.44 \%$ ) than at 1 week after planting (top view 3 dataset, average accuracy $=41.06 \%$ ), 9 weeks after planting (top view 2 dataset, average accuracy $=39.78 \%$ ), and top view dataset of several timepoint (top view dataset, average accuracy $=37.46 \%$ ). The final classification models used to classify each dataset were determined on the basis of 50 iterations with validation image sets and subsequently evaluated with independent data. The evaluation results showed that the accuracy of classification for the top view 1 dataset was $2.29 \%$ higher than that for the top view 2 dataset and $4.45 \%$ higher than that for the top view 3 dataset. These results indicated that similar developmental stages were shown 
better performance compared with different development stages (Supplementary Tables 9-11). In addition, more images are needed to construct classification models for various timepoint datasets. The prediction accuracies for the lateral view dataset were higher than those for the top view datasets. This is because the lateral view images showed diverse colors and more features than the top view images, such as the thickness of the leaves and the entire shape and stem of the plant.

The classification accuracies for the validation sets (step 1) and the test sets (step 2) depended on the type of architecture used (Figure 4). In the validation step, VGG19 showed the highest accuracy for the lateral view dataset, whereas GoogLeNet had the highest accuracy for the top view datasets. GoogLeNet showed the highest accuracy for all top view datasets in the test step. ResNet50 showed the lowest accuracy for all the datasets and was about $20 \%$ less accurate than GoogLeNet using the top view dataset in the test step. In previous studies, ResNet50 showed a relatively low performance to classify homogenous or highly similar images (Rudakov et al., 2018; Hassan et al., 2021). Therefore, the low accuracy of ResNet50 in our experiments might have been caused by a high degree of similarity among the images.

To investigate that hypothesis, we tested the architectures using pairs of accession types. The pairwise tests indicated that the classification of non-pekinensis together with any of the other three accession types was highly accurate, whereas that of the early introduced accessions was relatively inaccurate regardless of the other type of accession used. The classification accuracy for the Korean breeding accessions increased gradually depending on the other accession type in the pairwise test, with the early introduced accessions producing the lowest accuracy, the Chinese accessions producing higher accuracy, and the non-pekinensis accessions yielding the highest accuracy. According to the phylogenetic tree, the Korean breeding accessions are genetically close to the early introduced accessions and genetically distant from the non-pekinensis accessions (Figure 1). This suggests that phenotypic variances between the non-pekinensis accessions and the other accession types due to genetic dissimilarity led to high performance in the classification tests. The Korean breeding accessions were often classified accurately regardless of the deep learning architecture used, whereas the classifications of the Chinese accessions and early introduced accessions were inconsistent. These results further suggest that low accuracy in the classifications was caused by genetic differences between the accession types (Figure 1). Almost all of the early introduced accessions were imported from China in the early 1900s and have since been used as breeding sources. These accessions showed heterogenous phenotypes. On the other hand, the Korean breeding accessions have acquired homogenous phenotypes due to long-term breeding activities. In addition, the image depth for classification might affect the accuracy. The numbers of individual accessions used in the current analysis were not sufficient to train and evaluate deep learning models with very high accuracy for phenotypic classification. More images of various developmental stages would improve the accuracy of the models. Furthermore, tissue-specific and trait-specific images can be used in the future to identify trait-associated genes by correlation with genotypic data.

For applications of deep learning classification models in industrial fields, top view images are more suitable than lateral view images, although we achieved higher accuracy using the lateral view. Classification models like the ones presented in this study can be applied for nondestructive inspection of accessions and cultivars; however, improved models need to be developed, which can identify different types of plants with high accuracy using top view images.

\section{DATA AVAILABILITY STATEMENT}

The datasets presented in this study can be found in online repositories. The names of the repository/repositories and accession number(s) can be found in the article/Supplementary Material.

\section{AUTHOR CONTRIBUTIONS}

Y-MK conceived the project, designed the experiments, and organized the manuscript. MJ, JS, and SP performed the deep learning analysis. SH, SG, SK, and YL generated the phenotypic data. MJ and Y-MK wrote the manuscript. All authors contributed to the article and approved the submitted version.

\section{FUNDING}

This work was supported by the Korea Forest Service of the Korean government through its R\&D Program for Forestry Technology (Project No. 2014071H10-2122-AA04), and by the Korea Institute of Planning and Evaluation for Technology in Food, Agriculture, and Forestry through the Golden Seed Project (213006-05-5-SBG30) and the Technology Commercialization Support Program (821026-03), which is funded by a grant from the Ministry of Agriculture, Food, and Rural Affairs, the Ministry of Oceans and Fisheries, the Rural Development Administration, and the Korea Forest Service to Y-MK.

\section{SUPPLEMENTARY MATERIAL}

The Supplementary Material for this article can be found online in the Dryad repository via the DOIs indicated below.

Supplementary Figure 1 | Available via doi: 10.6084/m9.figshare.16620523.

Supplementary Figure 2 | Available via doi: 10.6084/m9.figshare.16620538.

Supplementary Figure 3 | Available via doi: 10.6084/m9.figshare.16620568.

Supplementary Figure 4 | Available via doi: 10.6084/m9.figshare.16620571.

Supplementary Figure 5 | Available via doi: 10.6084/m9.figshare.16620580.

Supplementary Figure 6 | Available via doi: 10.6084/m9.figshare.16620592.

Supplementary Figure 7 | Available via doi: 10.6084/m9.figshare.16620598.

Supplementary Tables 1-13 | Available via doi: 10.6084/m9.figshare.16620604. 


\section{REFERENCES}

Banzato, T., Bonsembiante, F., Aresu, L., Gelain, M., Burti, S., and Zotti, A. (2018a). Use of transfer learning to detect diffuse degenerative hepatic diseases from ultrasound images in dogs: a methodological study. Vet. J. 233, 35-40. doi: 10.1016/j.tvjl.2017.12.026

Banzato, T., Cherubini, G., Atzori, M., and Zotti, A. (2018b). Development of a deep convolutional neural network to predict grading of canine meningiomas from magnetic resonance images. Vet. J. 235, 90-92. doi: 10.1016/j.tvjl.2018.04.001

Bottou, L. (2010). "Large-scale machine learning with stochastic gradient descent," in Proceedings of COMPSTAT'2010 (Berlin: Springer), 177-186. doi: 10.1007/978-3-7908-2604-3_16

Chauhan, R., Ghanshala, K. K., and Joshi, R. (2018). "Convolutional neural network (CNN) for image detection and recognition," in 2018 First International Conference on Secure Cyber Computing and Communication (ICSCCC) (IEEE), 278-282.

Choi, Y., Lee, J., Park, D., and Chung, Y. (2018). Noise-robust porcine respiratory diseases classification using texture analysis and CNN. KIPS Trans. Softw. Data Eng. 7, 91-98. doi: 10.3745/KTSDE.2018.7.3.91

Dhaka, V. S., Meena, S. V., Rani, G., Sinwar, D., Ijaz, M. F., and Wozniak, M. (2021). A survey of deep convolutional neural networks applied for prediction of plant leaf diseases. Sensors 21:4749. doi: 10.3390/ s21144749

Grinblat, G. L., Uzal, L. C., Larese, M. G., and Granitto, P. M. (2016). Deep learning for plant identification using vein morphological patterns. Comput. Electron. Agric. 127, 418-424. doi: 10.1016/j.compag.2016. 07.003

Hao, X., Yang, G., Ye, Q., and Lin, D. (2019). "Rare animal image recognition based on convolutional neural networks," in 2019 12th International Congress on Image and Signal Processing, BioMedical Engineering and Informatics (CISP$B M E I$ ) (IEEE), 1-5.

Hassan, S. M., Maji, A. K., Jasiński, M., Leonowicz, Z., and Jasińska, E. (2021). Identification of plant-leaf diseases using $\mathrm{CNN}$ and transfer-learning approach. Electronics 10:1388. doi: 10.3390/electronics 10121388

He, K., Zhang, X., Ren, S., and Sun, J. (2016). "Deep residual learning for image recognition," in Proceedings of the IEEE Conference on Computer Vision and Pattern Recognition, 770-778.

Hedjazi, M. A., Kourbane, I., and Genc, Y. (2017). "On identifying leaves: a comparison of CNN with classical ML methods," in 2017 25th Signal Processing and Communications Applications Conference (SIU) (IEEE), 1-4.

Huang, G., Liu, Z., Van Der Maaten, L., and Weinberger, K. Q. (2017). "Densely connected convolutional networks." in Proceedings of the IEEE Conference on Computer Vision and Pattern Recognition, 4700-4708.

Iandola, F. N., Han, S., Moskewicz, M. W., Ashraf, K., Dally, W. J., and Keutzer, K. (2016). SqueezeNet: AlexNet-level accuracy with 50x fewer parameters and< $0.5 \mathrm{MB}$ model size. arXiv preprint arXiv:1602.07360.

Ingram, J. S., and Porter, J. R. (2015). Plant science and the food security agenda. Nat. Plants 1, 1-2. doi: 10.1038/nplants.2015.173

Jiang, Y., Li, C., Xu, R., Sun, S., Robertson, J. S., and Paterson, A. H. (2020). DeepFlower: a deep learning-based approach to characterize flowering patterns of cotton plants in the field. Plant Methods 16, 1-17. doi: 10.1186/s13007-020-00698-y

Kim, J. Y., Lee, H. E., Choi, Y. H., Lee, S. J., and Jeon, J. S. (2019). CNNbased diagnosis models for canine ulcerative keratitis. Sci. Rep. 9, 1-7. doi: 10.1038/s41598-019-50437-0

Kingma, D. P., and Adam, B. J. (2018). A method for stochastic optimization. 2014. arXiv preprint arXiv:1412.6980 9

Krizhevsky, A., Sutskever, I., and Hinton, G. E. (2012). Imagenet classification with deep convolutional neural networks. Adv. Neural Inf. Process. Syst. 25, 1097-1105. doi: 10.1145/3065386

Lecun, Y., Bottou, L., Bengio, Y., and Haffner, P. (1998). Gradient-based learning applied to document recognition. Proc. IEEE 86, 2278-2324. doi: $10.1109 / 5.726791$

Lee, S. H., Chan, C. S., Wilkin, P., and Remagnino, P. (2015). "Deep-plant: plant identification with convolutional neural networks," in 2015 IEEE International Conference on Image Processing (ICIP) (IEEE), 452-456.

Maeda-Gutierrez, V., Galvan-Tejada, C. E., Zanella-Calzada, L. A., Celaya-Padilla, J. M., Galván-Tejada, J. I., Gamboa-Rosales, H., et al. (2020). Comparison of convolutional neural network architectures for classification of tomato plant diseases. Appl. Sci. 10:1245. doi: 10.3390/app10041245

Mohammed, H. R., and Hussain, Z. M. (2021). Hybrid mamdani fuzzy rules and convolutional neural networks for analysis and identification of animal images. Computation 9:35. doi: 10.3390/computation 9030035

Pang, W., Li, X., Choi, S. R., Dhandapani, V., Im, S., Park, M. Y., et al. (2015). Development of a leafy Brassica rapa fixed line collection for genetic diversity and population structure analysis. Mol. Breed. 35, 1-15. doi: 10.1007/s11032-015-0221-9

Prajapati, S. A., Nagaraj, R., and Mitra, S. (2017). "Classification of dental diseases using CNN and transfer learning," in 2017 5th International Symposium on Computational and Business Intelligence (ISCBI) (IEEE), 70-74.

Ramchiary, N., Park, S., and Lim, Y. (2011). "Classical breeding and genetic analysis of vegetable Brassicas," in Genetics, Genomics and Breeding of Vegetable Brassicas, eds J. Sawdoski, C. Kole, 34-80.

Rudakov, N., Eerola, T., Lensu, L., Kälviäinen, H., and Haario, H. (2018). "Detection of mechanical damages in sawn timber using convolutional neural networks," in German Conference on Pattern Recognition (Springer), 115-126. doi: 10.1007/978-3-030-12939-2_9

Sardogan, M., Tuncer, A., and Ozen, Y. (2018). "Plant leaf disease detection and classification based on CNN with LVQ algorithm," in 2018 3rd International Conference on Computer Science and Engineering (UBMK) (IEEE), 382-385. doi: 10.1109/UBMK.2018.8566635

Shorten, C., and Khoshgoftaar, T. M. (2019). A survey on image data augmentation for deep learning. J. Big Data 6, 1-48. doi: 10.1186/s40537-019-0197-0

Simonyan, K., and Zisserman, A. (2014). Very deep convolutional networks for large-scale image recognition. arXiv preprint arXiv:1409.1556.

Szegedy, C., Ioffe, S., Vanhoucke, V., and Alemi, A. A. (2017). "Inception-v4, inception-resnet and the impact of residual connections on learning," in Thirtyfirst AAAI Conference on Artificial Intelligence.

Szegedy, C., Liu, W., Jia, Y., Sermanet, P., Reed, S., Anguelov, D., et al. (2015). "Going deeper with convolutions," in Proceedings of the IEEE Conference on Computer Vision and Pattern Recognition, 1-9.

Szegedy, C., Vanhoucke, V., Ioffe, S., Shlens, J., and Wojna, Z. (2016). "Rethinking the inception architecture for computer vision," in Proceedings of the IEEE Conference on Computer Vision and Pattern Recognition, 2818-2826.

Ubbens, J., Cieslak, M., Prusinkiewicz, P., and Stavness, I. (2018). The use of plant models in deep learning: an application to leaf counting in rosette plants. Plant Methods 14, 1-10. doi: 10.1186/s13007-018-0273-z

Wozniak, M., and Połap, D. (2018). Adaptive neuro-heuristic hybrid model for fruit peel defects detection. Neural Netw. 98, 16-33 doi: 10.1016/j.neunet.2017.10.009

Yudin, D., Sotnikov, A., and Krishtopik, A. (2019). "Detection of Big Animals on Images with Road Scenes using Deep Learning," in 2019 International Conference on Artificial Intelligence: Applications and Innovations (IC-AIAI) (IEEE), 100-1003.

Zeiler, M. D., and Fergus, R. (2014). "Visualizing and understanding convolutional networks," in European Conference on Computer Vision (Springer), 818-833.

Conflict of Interest: Author MJ, JS, and JP are employed by Euclidsoft Co., Ltd.

The remaining authors declare that the research was conducted in the absence of any commercial or financial relationships that could be construed as a potential conflict of interest.

Publisher's Note: All claims expressed in this article are solely those of the authors and do not necessarily represent those of their affiliated organizations, or those of the publisher, the editors and the reviewers. Any product that may be evaluated in this article, or claim that may be made by its manufacturer, is not guaranteed or endorsed by the publisher.

Copyright (c) 2021 Jung, Song, Hong, Kim, Go, Lim, Park, Park and Kim. This is an open-access article distributed under the terms of the Creative Commons Attribution License (CC BY). The use, distribution or reproduction in other forums is permitted, provided the original author(s) and the copyright owner(s) are credited and that the original publication in this journal is cited, in accordance with accepted academic practice. No use, distribution or reproduction is permitted which does not comply with these terms. 